\title{
Image Stitching Method Based on Genetic Algorithm
}

\author{
Yang $\mathrm{Xu}$, Changbo Sun
}

Chongqing University of posts and telecommunications, Chongqing 400065, China; Chongqing University of posts and telecommunications, Chongqing 400065, China; 446588010@qq.com, 1303291264@qq.com

Keywords: image stitching, genetic algorithm, image fusion, RANSAC, Laplacian pyramid

\begin{abstract}
In allusion to the inaccuracy of homography matrix using RANSAC from image stitching based on image features, a new image stitching algorithm based on genetic algorithm was proposed. First, locate and describe the feature points of images using feature extraction algorithm, and and the coarse matching was performed based on the ratio between nearest distance and next nearest distance. Then get optimal transform model using genetic algorithm. At last, use the Laplacian transform pyramid reconstruction algorithm to reconstruct and obtain the final fused image. The experiment show that compared with common methods, the method proposed in this paper performs better in terms of accuracy of homography matrix. Subjectively, it improves the quality and effect of fusion image.
\end{abstract}

\section{Introduction}

Image stitching technology is a technology that stitches images which have overlapping regions to a wide-angle,no-gap and high resolution panorama ${ }^{[1]}$.The bigger shooting angle ,the lower image resolution when use general camera to get wide-angle image ${ }^{[2]}$.And panoramic camera or wide-angle lens not only is expensive but also have relatively higher image distortion.In order to get image with wider even 360 view under the condition that not bring down the resolution of image,the image stitching technology by means of computer devices appeared and developed like a raging fire ${ }^{[3,4]}$.

Image registration is an important subject in image stitching.Image registration is defined as the process that matching and overlay two or more images which describe the same scene and collected in different time,different perspectives and by different transducer ${ }^{[5]}$. There are two kinds of image registration methods sort by realization forms:registration based on image gray scale and registration based on image features ${ }^{[6]}$. The registration based on image gray scale directly uses image gray scale to compute similarity measure between two images and uses some searching means to search the transformation model parameters which make similarity measure maximum or minimum ${ }^{[7]}$.The registration based on image features firstly extract mutual features between two images and then matches these features and gets geometric transformation model parameters to realize image registration ${ }^{[8]}$.The registration based on image features compresses information capability,operates rapidly,robust to the gray level transformation,is used widely.Feature corner is the ideal image characteristics, the coordinate of feature corner can be directly used to estimate image geometric transformation model, so registration method based on feature corner is research hotspot in recent years $^{[9]}$.

The registration based on image features generally uses SIFT(Scale Invariant Feature Transform) or SURF(Speed Up Robust Features) to extract the feature corner from the images.Roughly matches these feature corners,and then uses RANSAC (Random Sampling Consensus) matching algorithm to reject mismatching points and get image transformation model finally ${ }^{[10]}$.However, when the amount of rough matching points is less or existing rotation of angle between images,such method will lead to transformation model inaccuracy.Aim at above problem,this paper raised a method based on genetic algorithm to estimate the optimal transformation model,then use Laplacian Pyramid algorithm to fuse and stitch these registered images ${ }^{[11]}$. 


\section{Feature Point Detection and Description Based on SURF Algorithm}

SURF algorithm is improved and accelerated based on SIFT algorithm,robust to the scale and rotation.Feature points detection of SURF algorithm is based on the theory of scale space,it uses the determinant of Hessian matrix as discriminant to detect local maximum values.

The Hessian matrix at some point where scale is $\delta$ is defined as:

$$
H(u, \sigma)=\left[\begin{array}{ll}
I_{x x}(u, \sigma), & I_{x y}(u, \sigma) \\
I_{y x}(u, \sigma), & I_{x x}(u, \sigma)
\end{array}\right]
$$

In the formula, $I_{x x}(u, \sigma) 、 I_{x y}(u, \sigma)$ and $I_{y y}(u, \sigma)$ is defined as convolution of second derivative of Gaussian function and image at point $u$ The determinant of Hessian matrix is defined as:

$$
\operatorname{det}(H)=I_{x x}(u, \sigma) * I_{y y}(u, \sigma)-I^{2}{ }_{x y}(u, \sigma)
$$

SURF algorithm uses grid type filter to approximate instead of second derivative of Gaussian function,it makes convolution operation speed up.Grid type filter which size is $9 * 9$ as shown in Fig.1.The three images above are second derivative of Gaussian function in different directions.The three images below are approximate grid type filter,respectively labeled as $D_{x x}, D_{y y}$ and $D_{x y}$.

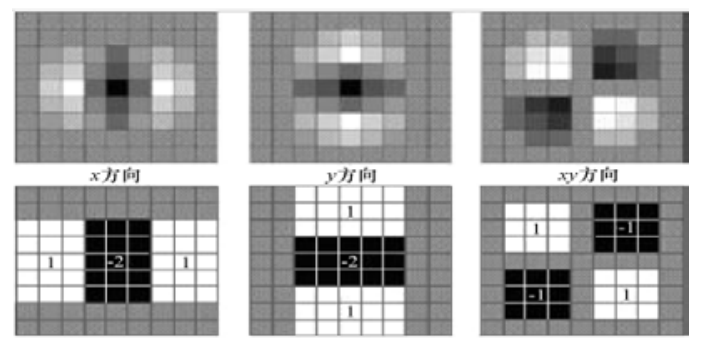

Fig. 1 Approximate representation of Gaussian second derivative

Use this filter,the approximate expression of formula 2 is as follow:

$\operatorname{det}(H)=D_{x x} * D_{y y}-\left(0.9 * D_{x y}\right)^{2}$

After establishing multiscale image pyramid,detecting feature points at different scales, accurately locating feature points,steady feature points can be gotten. These feature points need features description after be located.The construction of SURF feature points descriptor consists of two steps:allocate principal direction and produce feature vector,both of them are proceeding at scale $\delta$.Use feature point as the center and $6 \delta$ as radius to form a circle,get Haar wavelet response factor in $\mathrm{x}$ and $\mathrm{y}$ direction inside the circle,calculate the sum of factor in $\mathrm{x}$ and $\mathrm{y}$ direction inside a $60^{\circ}$ sector,and structure a new Vector.Rotate the sector to traverse the circle,use the direction of the longest vector as principal direction.Center on feature points,rotate coordinate axis to principal direction,and divide the square window center on feature points into $4 * 4$ child-windows.Take $\delta$ as sample interval,calculate wavelet response in the $\mathrm{x}$ and $\mathrm{y}$ direction within the child window,and labeled as $d x$ and $d y$.

As shown in Fig.2,accumulate the coefficient inside the child window to form a four-dimensional vector $V=\left[\sum d_{x}, \sum d_{y}, \sum\left|d_{x}\right|, \sum\left|d_{y}\right|\right]$.All vectors of child windows make up of feature point description vector which have $4 * 4 * 4=64$ dimensions

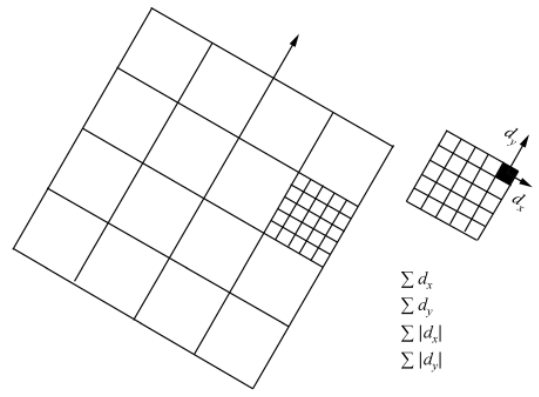

Fig 2 Constitution of feature descriptor 


\section{Feature points coarse matching}

Feature points should be matched after be detected,thus establish correspondence of the same physical location.Assume that $P_{A}$ is a discretionary feature point of image $\mathrm{A}, P_{B}$ is a discretionary feature point of image B,their feature point description vectors are $\operatorname{Descr}_{A}$ and $\operatorname{Descr}_{B}$ respectively, $\operatorname{Descr}_{A i}$ and $\operatorname{Descr}_{B i}$ are their i-th component ,

The distance between $P_{A}$ and $P_{B}$ is defined as:

$$
D\left(P_{A}, P_{B}\right)=\sqrt{\sum_{i=1}^{n}\left(\text { Descr }_{A i}-\text { Descr }_{B i}\right)^{2}}
$$

ND represents nearest distance and NND represents second rate nearest distance,thus has the ratio of distance $R=N D / N N D$.Suppose $\mathrm{T}$ is a positive number which less than 1,there is following formula:

$\begin{cases}\text { if } R \leq T & \text { success } \\ \text { else } & \text { failure }\end{cases}$

Experiment shows $T \in[0.5,0.7]$ is a ideal range.The essential methodology of this operation is that taking as this feature point as matching point only if the nearest distance has clear advantage over the second rate nearest distance.

\section{Estimation of transformation model based on Genetic algorithm}

Genetic algorithm (GA) is a random search method based on the biological laws of evolution.It is first proposed in 1975 by professor J.Holland,Its main characteristics include operating directly on the object structure,derivation and function continuity is not necessary;Having intrinsic concurrency and better performance in the abilities of seeking the global excellent result.

The operations of Genetic algorithm include selection,crossover and mutation,be used to simulate reproduction,hybridize and mutation in the process of natural selection and mutation.Genetic algorithm starts with the population which represents the potential solution set of a problem,and then map phenotype to genetic code.Every genetic code represents a solution of the problem,called chromosome or individual.Start from the initial population,evolve from generation to generation thus generate the population which fitness is higher and higher.In each generation,selecting individual according to fitness and mutation operation and crossover operation on the basis of principles of genetics are used on the current population,generating a new population which represents the new solution set of the problem.Just as evolution principle,the offspring population will has higher fitness than the previous population.Decode the optimal individual in the last population and then get the optimal solution of the problem.

The steps of seeking optimal transformation model using genetic algorithm in this paper as follows:

Step 1:Create the initial generation.Random select 4 feature point matching pairs from the coarse matching pairs,gain transformation model and code the parameters of the model using binary system.Combine these binary coding orderly and get a new chromosome or individual.Set the count of all feature point matching pairs to $\mathrm{M}$, and the the count of feature point matching pairs conform to this transformation model to $\mathrm{N}$, then set $P=N / M$ to the fitness of this individual.Repeat the above process and get 200 individuals.Select 100 individuals which have higher fitness from the 200 individuals as the initial generation,this makes the all individuals in the initial generation have higher fitness,speed up the evolution and get the optimal solution faster.

Step 2:replication.Replicate the individuals which have higher fitness with a certain probability to next generation,thus weed out the individuals which have lower fitness.Produce next generation on the basis of the corresponding feature point matching pairs of last generation,and serve $P=N / M$ as fitness function.The individual amount of the new generation remain unchanged,but the average fitness become higher. 
Step 3:crossover.Getting a new individual from two individuals in the population through decomposition and combination,which intersects the binary codings of individuals to simulate the process of crossover in this paper.Not all new individuals came from crossover ,used a probability to control the amount of individuals which execute operation of crossover,and the higher individual fitness, the lower probability of crossover,thus made gene coding of individual which has high fitness remain unchanged.

Step 4:mutation.Occasional and randomly change a parameter of gene coding in the population to simulate the process of mutation.It plays a supporting role in genetic algorithm.

Step 5:Sort these individuals on the basis of fitness.

Step 6:Stop the process if the highest fitness of individual have not been increased markedly.The individual which has the highest fitness in the generation denotes the optimal solution.Otherwise,skip ahead to step 2.

Step 7:Decode the optimal solution to get the transformation model.

Genetic algorithm is not blind searching,also is not brute-force searching,it proceeds guided searching according to the living environment of individuals.It only depends on the value of target,therefore has extensive universality.The operand of the algorithm is a group of individuals ,instead of single individual, has multistrip search trajectories,thus has covert parallelism.

\section{Image fusion based on Laplacian Pyramid}

In allusion to the problems that losing details and result influenced by deviation and noise in Laplacian Pyramid image fusion algorithm,the image fusion algorithm based on activity degree and matched-degree was introduced in this paper.The algorithm process as Fig.3.

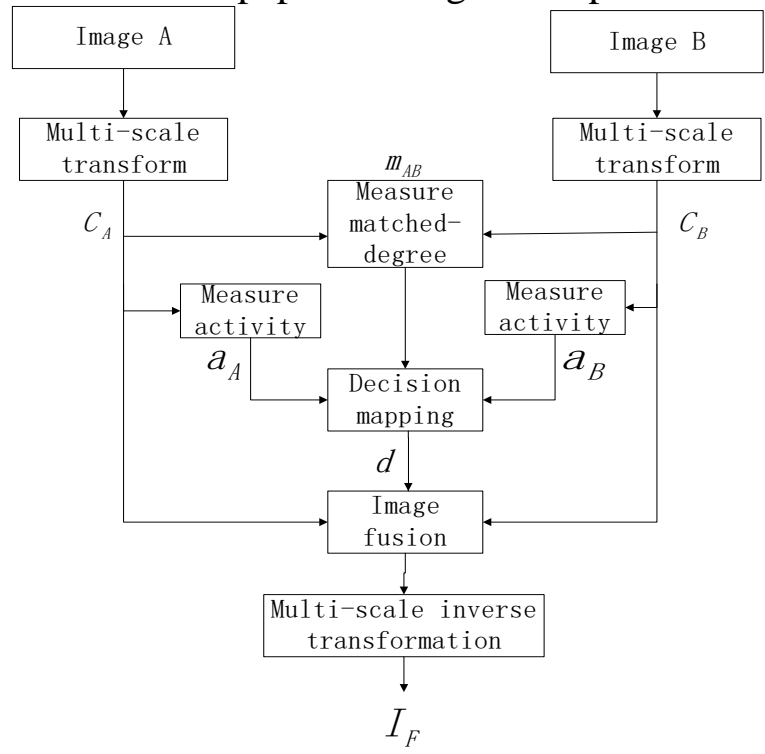

Fig. 3 Fusion process

Activity is used to measure the significance level of multi-scale transform coefficient.Activity has multiple definitions according to different fusion task.Local window energy was used as activity in this paper,defined as follow:

$$
a_{s}^{j, k}=\sum_{\left(x^{\prime}, y^{\prime}\right) \in P}\left|C_{s}^{j, k}\left(x+x^{\prime}\right)\left(y+y^{\prime}\right)\right|
$$

In the formula, $C_{s}^{j, k}$ is the sub-band coefficient of image at scale $j$ and direction $k$,and $P$ represents window scope.

Matched-degree is used to measure the level of similarity between images,is defined as the normalized cross-correlation of local window coefficients: 


$$
m_{A B}^{j, k}(x, y)=\frac{2 \sum_{\left(x^{\prime}, y^{\prime}\right) \in P} w\left(x^{\prime}, y^{\prime}\right) C_{A}^{j, k}\left(x+x^{\prime}, y+y^{\prime}\right) C_{B}^{j, k}\left(x+x^{\prime}, y+y^{\prime}\right)}{\sum_{\left(x^{\prime}, y^{\prime}\right) \in P} w\left(x^{\prime}, y^{\prime}\right)\left(\left|C_{A}^{j, k}\left(x+x^{\prime}, y+y^{\prime}\right)\right|^{2}+\left|C_{B}^{j, k}\left(x+x^{\prime}, y+y^{\prime}\right)\right|^{2}\right)}
$$

In the formula, $w\left(x^{\prime}, y^{\prime}\right)$ represents weight of window and $m_{A B}^{j \cdot k}(x, y)$ represents matched-degree at location $(x, y)$.

Get the decision value according to activity and matched-degree:

$$
d^{j, k}(x, y)=\left\{\begin{array}{c}
1, \text { if } m_{A B}^{j, k}(x, y)<T \text { and } a_{A}^{j k}(x, y) \geq a_{B}^{j k}(x, y) \\
0, \text { if } m_{A B}^{j, k}(x, y)<T \text { and } a_{A}^{j k}(x, y)<a_{B}^{j k}(x, y) \\
1 / 2, \text { others }
\end{array}\right.
$$

In the formula, $\mathrm{T}$ represents threshold,general assigned from 0.7 to 0.8 .

The decision value was used to guide the fusion of different layers in Laplacian Pyramid.This process can be described as follow:

$I_{F}=\alpha_{A} L_{A}+\alpha_{B} L_{B}$

In above formula, $\alpha_{A}=1-\alpha_{B}=d^{j, k}(x, y)$.

At last,reconstruct image from up to down according to the fused Laplacian Pyramid,and get the stitched image.

\section{Experiment results and analysis}

Extracted feature points from input image and reference image, and there were 3517 feature points in input image and 3258 feature points in reference image.Remained 395 feature points matching pairs after coarse matching,as shown in Fig 4.As can be seen from figure 4,there were much mismatching after coarse matching.Remained 236 feature points matching pairs after rejecting mismatching pairs by RANSAC algorithm, as shown in figure Fig 5.As can be seen from Fig 5,some mismatching pairs were rejected after using RANSAC algorithm,however,minority mismatching pairs still existting in these matching pairs.Using the algorithm of this paper,remained 165 matching pairs which conform to the transformation model.As shown in Fig 6,the algorithm of this paper refined these matching pairs as can as possible,improved the accuracy of image rectification.The stitched image as shown in Fig 7.

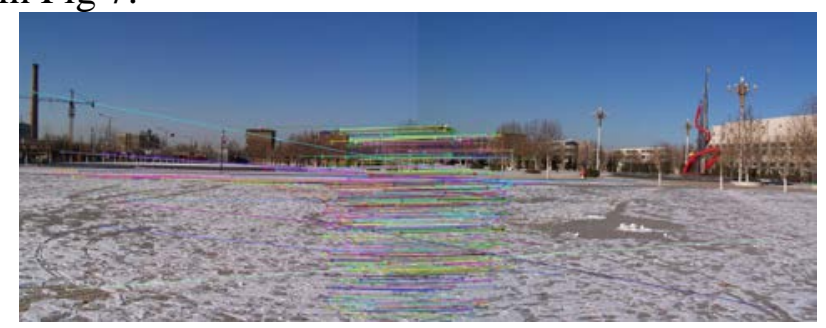

Fig 4 Matching pairs after coarse matching

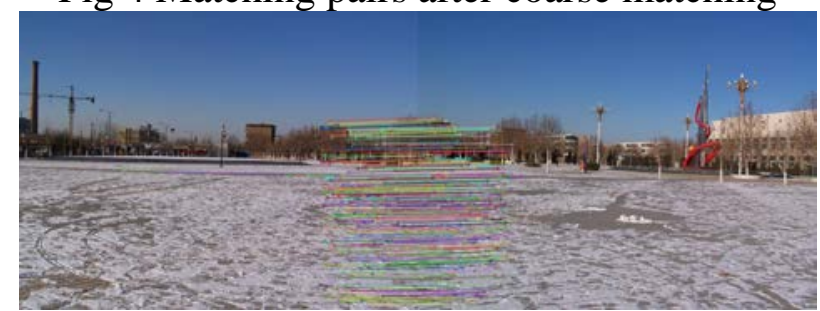

Fig 5 Matching pairs after using RANSAC 


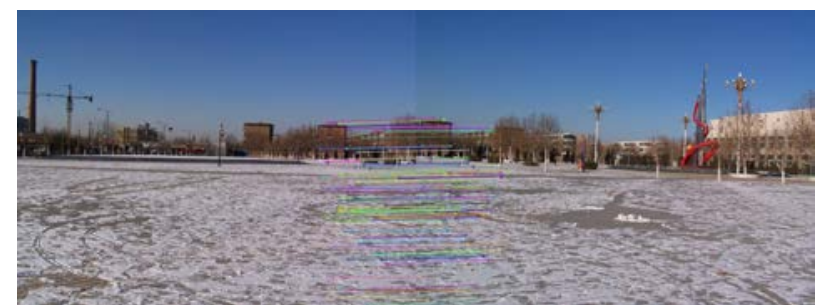

Fig 6 Matching pairs conform to the transformation model in this paper

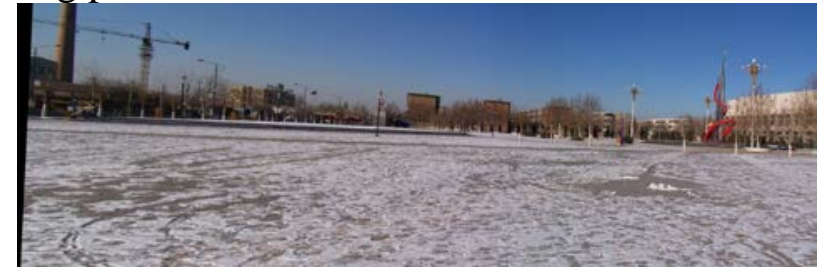

Fig7 Stitching image of the algorithm in this paper

Adopted 5 groups images,used RANSAC algorithm and algorithm of this paper to refine feature points matching pairs,and used standard deviation and information entropy to evaluate result,the final data are shown in table 1.Standard deviation reflects the dispersion degree of image gray value relative to average gray value.The bigger the gray value,the higher the image quality.Information entropy is a measurement of the image average information content.As can be seen from table 1,compared with RANSAC algorithm, the algorithm in this paper can rejected mismatching pairs as can as possible on the basis of guaranteeing the amount of matching pairs,and obviously improves standard deviation and information entropy,has better stitching result.

Table 1 Experiment data contrast

\begin{tabular}{|c|c|c|c|c|c|}
\hline & $\begin{array}{c}\text { Group } \\
1\end{array}$ & Group 2 & Group 3 & Group 4 & Group 5 \\
\hline $\begin{array}{c}\text { Matching pairs after } \\
\text { course matching }\end{array}$ & 395 & 338 & 451 & 215 & 359 \\
\hline RANSCA & 236 & 230 & 308 & 176 & 230 \\
\hline Genetic algorithm & 165 & 145 & 213 & 117 & 137 \\
\hline $\begin{array}{c}\text { Standard deviation } \\
\text { of RANSCA } \\
\text { matching }\end{array}$ & $\begin{array}{c}60.125 \\
6\end{array}$ & 55.3236 & 58.1259 & 50.8768 & 53.1258 \\
\hline $\begin{array}{c}\text { Standard deviation } \\
\text { of matching based } \\
\text { on Genetic } \\
\text { algorithm }\end{array}$ & $\begin{array}{c}63.568 \\
2\end{array}$ & 58.6795 & 62.5869 & 53.4987 & 55.6874 \\
\hline $\begin{array}{c}\text { Information entropy } \\
\text { of RANSAC } \\
\text { matching }\end{array}$ & 8.3286 & 8.1257 & 9.1586 & 7.4887 & 7.8657 \\
\hline $\begin{array}{c}\text { Standard deviation } \\
\text { of matching based } \\
\text { on Genetic } \\
\text { algorithm }\end{array}$ & 9.6895 & 9.3359 & 10.5681 & 8.6795 & 8.9247 \\
\hline
\end{tabular}

As can be seen from experimental data,by using the algorithm in this paper, stitching image can be matched more accurately,stitching result is favorable.

\section{Conclusions}

Fast and robust SURF algorithm was used in this paper to extract the feature points,after coarse matching,used genetic algorithm which rejects mismatching pairs effective and improves the matching accuracy to get the transformation model of stitching images,at last,the fusion method 
based on Laplacian Pyramid was used to reconstruct image. The result of experiments show that the algorithm in this paper can achieve benign stitching result.

\section{Acknowledgments}

This work was financially supported by the engineering study and industrialization of car networking intelligent information terminal fund (KJZH14207).

\section{References}

[1]Szeliski R. Image alignment and stitching: a tutorial[J]. Foundations \& Trend@ in Computer Graphics \& Vision, 2006, 2(1):1-104.

[2]Wang Z, Ziou D, Armenakis C, et al. A comparative analysis of image fusion methods[J]. IEEE Transactions on Geoscience \& Remote Sensing, 2012, 43(6):1391-1402.

[3]Jia J, Tang C K. Image Stitching Using Structure Deformation[J]. IEEE Transactions on Pattern Analysis \& Machine Intelligence, 2008, 30(4):617-31.

[4]Zitová B, Flusser J. Image registration methods: a survey[J]. Image \& Vision Computing, 2003, 21(11):977-1000.

[5]Ashburner J. A fast diffeomorphic image registration algorithm[J]. Neuroimage, 2007, 38(1):95-113.

[6]Lagae A, Drettakis G. Filtering solid Gabor noise[J]. Acm Transactions on Graphics, 2011, 30(4):51:1-51:6.

[7]Huang W, Han X. An Improved RANSAC Algorithm of Color Image Stitching[J]. Lecture Notes in Electrical Engineering, 2013, 256:21-28.

[8]Bay H, Ess A, Tuytelaars T, et al. Speeded-Up Robust Features (SURF)[J]. Computer Vision \& Image Understanding, 2008, 110(3):346-359.

[9]Michalewicz Z. Genetic Algorithms + Data Structures = Evolution Programs[J]. 2015.

[10]Rudolph G. Convergence analysis of canonical genetic algorithm. IEEE Trans Neural Netw[J]. IEEE Transactions on Neural Networks, 1994, 5(1):96-101.

[11]Loza A, Bull D, Canagarajah N, et al. Non-Gaussian model-based fusion of noisy images in the wavelet domain[J]. Computer Vision \& Image Understanding, 2010, 114(1):54-65. 